\title{
Prophylactic Level VII Nodal Dissection as a Prognostic Factor in Papillary Thyroid Carcinoma: a Pilot Study of 27 Patients
}

\author{
Ihab Samy Fayek
}

\begin{abstract}
Background: Prognostic value of prophylactic level VII nodal dissection in papillary thyroid carcinoma has been highlighted. Materials and Methods: A total of 27 patients with papillary thyroid carcinoma with N0 neck underwent total thyroidectomy with level VI and VII nodal dissection through same collar neck incision. Multicentricity, bilaterality, extrathyroidal extension, level VI and VII lymph nodes were studied as separate and independent prognostic factors for DFS at 24 months. Results: 21 females and 6 males with a mean age of 34.6 years old, tumor size was $5-24 \mathrm{~mm}$. (mean $12.4 \mathrm{~mm}$.), multicentricity in 11 patients 2-4 foci (mean 2.7), bilaterality in 8 patients and extrathyroidal extension in 8 patients. Dissected level VI LNs 2-8 (mean 5 LNs) and level VII LNs 1-4 (mean 1.9). Metastatic level VI LNs 0-3 (mean 1) and level VII LNs 0-2 (mean 0.5). Follow-up from 6-51 months (mean 25.6) with 7 patients showed recurrence (3 local and 4 distant). Cumulative DFS at 24 months was $87.8 \%$ and was significantly affected in relation to bilaterality (p-value $<0.001$ ), extrathyroidal extension (p-value $<0.001)$, level VI positive ((p-value $<0.001)$ and level VII positive ((p-value $<0.001)$ LNs. No recurrences were detected during the follow-up period in the absence of level VI and level VII nodal involvement. Conclusions: Level VII prophylactic nodal dissection is an important and integral prognostic factor in papillary thyroid carcinoma. A larger multicenter study is crucial to reach a satisfactory conclusion about the necessity and safety of this approach.
\end{abstract}

Keywords: Level VII - central nodal dissection - papillary thyroid carcinoma

Asian Pac J Cancer Prev, 16 (10), 4211-4214

\section{Introduction}

Papillary thyroid cancer (PTC) is the most common type of thyroid cancer accounting for about $80 \%$ of all thyroid cancers, and is the fifth leading malignancy in females (Cisco et al., 2012; Siegel et al., 2013).

Indications for lymphadenectomy "on principle" or "necessary" in the treatment of PTC are the subject of lively debate in literature. Several authors wrote of their experiences but there isn't any prospective randomized trial decisive contribution to the discussion (Carling et al., 2012).

PTC presents a high incidence of lymph-node metastases, particularly in the central compartment (level VI). The presence of clinically evident lymph node metastases in the central compartment requires, besides total thyroidectomy (TT), also central neck dissection (CND). CND carried out (clinically or availing of radiology) to remove apparent metastatic lymph-nodes in the central compartment $(\mathrm{cN} 1 \mathrm{a})$ is called "therapeutic central neck dissection" while central lymphadenectomy performed in the absence of suspected lymph-node metastases (cNO) is defined "prophylactic" or "elective" (Sobin et al., 2010).
Indication for prophylactic $\mathrm{CND}$ in $\mathrm{CN} 0$ appears to be correlated with incidences of postoperative complications. Dralle (2012) reports a significant risk of postoperative hypoparathyroidism after CND, but suggests that prophylactic central lymph node dissection improves prognosis for papillary thyroid cancer Dralle (2013).

A recent systematic review of imaging for central neck LN involvement in PTC found poor sensitivity for both ultrasound and CT. The study demonstrated the sensitivity of ultrasound and CT to range between 50 and $70 \%$ Mulla, (2012).

The American Thyroid Association's (ATA) management guidelines on PTC specify a CND to be a level VI dissection only (recommendation 27) (Cooper et al., 2009) Alternatively, the ATA's consensus statement on terminology of CND defines the innominate artery as the lower limit of a CND (ATA consensus, 2009) This second definition equates CND to level VI and the superior portion of level VII. To add to the confusion, the AJCC's TMN classification of PTC considers level VII nodes to be regional nodes, carrying the same prognosis as lateral neck lymph nodes.

According to Mazzaferri et al. (2009) the incidence of transient hypoparathyroidism after CND ranges between 
Ihab Samy Fayek

$14 \%$ and $44 \%$. For White et al., 2007 the rate of permanent hypoparathyroidism after total thyroidectomy (TT) with prophylactic CND is between $0 \%$ and $14.3 \%$.

Many studies showed an increased risk of recurrent paralysis in patients undergoing CND, with rates of recurrent lesions ranging between $1 \%$ and $12 \%$ (Hughes and Doherty, 2011).

According to Popadich et al. (2011) the percentage of recurrent transient paralysis increased from $1.8 \%$ after TT to $2.3 \%$ after TT with CND, whereas the definitive rates range from $0.4 \%$ to $1.8 \%$. For Giordano et al., 2012 transitional cases range from $3.6 \%$ to $5.5 \%$, the definitive from $1 \%$ to $2.3 \%$.

This pilot study was designed to study the prognostic value of prophylactic level VII nodal dissection in PTC and its importance among other prognostic factors.

\section{Materials and Methods}

From November 2009 to April 2013, 27 patients diagnosed with papillary thyroid carcinoma (confirmed by U/S guided FNAC) underwent total thyroidectomy and prophylactic nodal dissection of the central neck nodes (level VI) in addition to the superior mediastinal nodes (level VII) all through the low collar neck incision.

All patients had no clinical nor sonographic evidence of nodal metastases pre-operatively. The limits of dissection was the hyoid bone (above), the left innominate vein (below) and the internal jugular veins on both sides (laterally).

The 3 specimens were submitted intraoperatively in separate containers for histopathological examination as follows:

i) Total thyroidectomy specimen; ii) Level VI group of lymph nodes (including the prelaryngeal, pretracheal, and paratracheal nodes between the hyoid bone and the suprasternal notch); iii) Level VII group of lymph nodes (defined as the pretracheal and paratracheal superior mediastinal lymph nodes between the suprasternal notch and the innominate vein).

Patient demographics, number of nodes removed, percentage of nodes involved by malignancy, and size of the metastases were recorded and analyzed. A single pathologist reviewed all histology of patients. This project was approved by the Institutional Ethics Committee.

Data were analyzed using IBM SPSS Advanced Statistics version 20.0 (SPSS Inc., Chicago, IL). Chisquare test (Fisher's exact test) was used to examine the relation between qualitative variables. Survival analysis was done using the Kaplan-Meier method and comparison between two survival curves was done using log-rank test. A p-value $<0.05$ was considered significant.

\section{Results}

Twenty one females and six males were included in the study, the age was 22-51 years old (mean 34.6). Tumor size was 5-24 mm. in maximum diameter (mean 12.4 $\mathrm{mm}$.). Multicentricity was observed in 11 patients with 2-4 foci (mean 2.7) where two foci in 5 patients $(45.5 \%)$, three foci in 4 patients $(36.4 \%)$ and four foci in 2 patients
$(18.2 \%)$. Bilaterality was observed in 8 patients $(29.6 \%)$. Extrathyroidal extension was also observed in 8 patients (29.6\%). The number of dissected level VI lymph nodes ranged from 2-8 LNs (mean $5 \mathrm{LNs}$ ) and the number of dissected level VII lymph nodes ranged from 1-4 LNs (mean 1.9 LN). The number of metastatic level VI lymph nodes ranged from 0-3 LNs (mean $1 \mathrm{LN}$ ) and The number of metastatic level VII lymph nodes ranged from 0-2 LNs

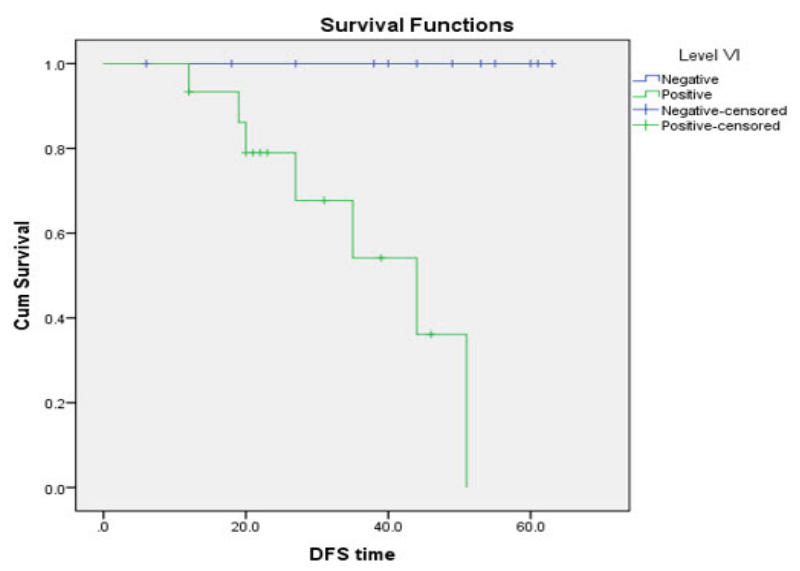

Figure 1. Correlation between Cumulative Disease Free Survival and Level VI Nodes

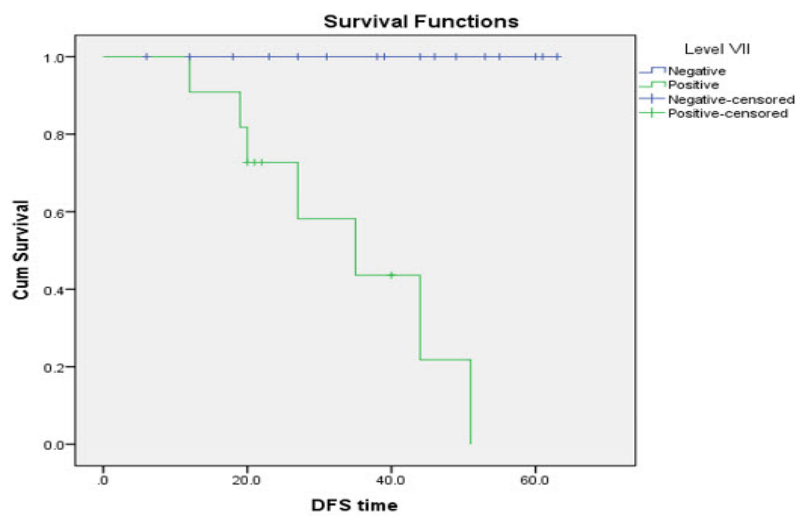

Figure 2. Correlation between Cumulative Disease Free Survival and Level VII Nodes

Table 1. Cumulative DFS at 24 Months for the Study Population in Relation to the Prognostic Factors

\begin{tabular}{lccc}
\hline & Number & $\begin{array}{c}\text { Cumulative Disease } \\
\text { Free Survival }\end{array}$ & -value \\
\hline All cases & 27 & $87.80 \%$ & \\
Multicentricity & & & 0.004 \\
$\quad$ Yes & 11 & $81.80 \%$ & \\
$\quad$ No & 16 & $92.30 \%$ & $<0.001$ \\
Bilaterality & & & \\
$\quad$ Yes & 8 & $75.00 \%$ & $<0.001$ \\
$\quad$ No & 19 & $93.80 \%$ & \\
Extrathyroidal Extension & & \\
$\quad$ Yes & 8 & $62.50 \%$ & $<0.001$ \\
$\quad$ No & 19 & $100 \%$ & \\
Level VI lymph nodes & & & \\
$\quad$ Positive & 15 & $79.00 \%$ & \\
$\quad$ Negative & 12 & $100.00 \%$ & \\
Level VII lymph nodes & & $72.70 \%$ & \\
$\quad$ Positive & 11 & $100 \%$ & \\
Negative & 16 & &
\end{tabular}


(mean 0.5 LN). Temporary hypoparathyroidism, with a serum calcium concentration of less than $8 \mathrm{mg} / \mathrm{dL}$ at any point after the operation, developed in 10 patients (37\%) and only 2 patients $(7.4 \%)$ were in need for calcium or vitamin D supplementation at 6 months after the operation (Permanent hypoparathyroidism). Temporary vocal cord dysfunction was defined as decreased or absent vocal cord motility postoperatively on direct laryngoscopy that resolved subsequently was observed in 4 patients (14.8\%) with only one patient $(3.7 \%)$ had permanent recurrent laryngeal dysfunction with the absence of vocal cord motility on direct laryngoscopy persisting beyond 6 months after the operation. Follow-up period ranged from 6-51 months (mean 25.6) with 7 (25.9\%) patients showed recurrences ( 3 local and 4 distant). No recurrences were diagnosed during the follow up period in patients with absent level VI and level VII nodal involvement in the study. The cumulative disease free survival at 24 months was significantly affected by multicentricity ( $p$ value $=0.004)$, bilaterality $(p$ value $<0.001$ ), extrathyroidal extension ( $\mathrm{p}$-value $<0.001$ ), level VI positive (p-value $<0.001$ ) (Figure 1) and level VII positive (p-valu <0.001) LNs (Figure 2) (Table 1).

\section{Discussion}

Putting in mind that in PTC, lymph node metastases have been shown to increase the risk of locoregional tumor recurrence (Hay et al., 1999; Leboulleux et al., 2005; Cheema et al., 2006; Ito and Miyauchi, 2007) and that approximately $5 \%$ of patients with PTC were identified as having superior mediastinal metastases (Roh et al., 2008; Machens and Dralle, 2009) with concerns that metastatic lymph node involvement is nothing more than indolent microscopic disease without clinical significance and that the sensitivity of ultrasound and CT range between only 50 and $70 \%$ Mulla,(2012), and on the other hand Wang et al., 2013 advocated the unnecessary CND in some patients with incidental PTC postoperatively and that the impact of positive regional lymph nodes on survival has been controversial (Steinmuller et al., 2000), we performed a prophylactic CND including level VI and VII from the same collar neck incision (Block et al., 1972). Yu et al., 2012 recommended the "wait and see" strategy for clinically negative level V in low risk PTC patients and stated that it would still achieve good survival results. As is recommended for the other nodal groups, dissection of the superior mediastinal lymph nodes should it be performed in the presence of gross nodal involvement? Some investigators advocate for routine prophylactic superior mediastinal dissection in patients with PTC; however, data indicating a survival or recurrence advantage are lacking (Fritze and Doherty, 2010). In this study, a DFS advantage at 24 months was statistically significant in patients with negative metastases to levels VI and VII lymph nodes; in addition, no recurrences were detected neither clinically nor radiologically in all those patients during the followup period which reached up to 51 months. These results suggests the importance of dissecting these nodes, they also re-enforce and support the study of Laura et al., 2013 who performed prophylactic Level VI and VII nodal dissection on 32 patients with PTC concluding the importance and safety of level VII nodal dissection and that failure to include this level will leave significant macrometastatic nodal disease in situ.

Inspite the low incidence of permanent hypoparathyroidism $(7.4 \%)$ and permanent recurrent laryngeal dysfunction $(3.7 \%)$ in this study, which is comparable to other studies in the literature (White et al., 2007; Popadich et al., 2011; Giordano et al., 2012), we cannot conclude the safety of the procedure as regards those two parameters as this may need a larger sample size in a multicenter study with a multivariate analysis comparing another group of patients who didn't have a prophylactic Level VII nodal dissection.

In conclusion, level VII (as well as level VI) prophylactic nodal dissection is an important prognostic indicator in PTC and should be performed in all patients with clinically and radiologically N0 neck. A multivariate analysis along a multicenter study with a larger number of patients must be performed to reach a satisfactory conclusion about the necessity and safety of level VII nodal dissection.

\section{References}

American Thyroid Association Surgery Working, Carty S, Cooper D, Doherty G, et al (2009). Consensus statement on the terminology and classification of central neck dissection for thyroid cancer. Thyroid, 19, 1153-8.

Block M, Miller J, Horn R (1972). Significance of mediastinal lymph node metastases in carcinoma of the thyroid. Am J Surg, 123, 702-5.

Carling T, Carty S, Ciarleglio M, et al (2012). American thyroid association surgical affairs committee. American thyroid association design and feasibility of a prospective randomized controlled trial of prophylactic central lymph node dissection for papillary thyroid carcinoma. Thyroid, 22, 237-44.

Cheema Y, Repplinger D, Elson D, et al (2006). Is tumor size the best predictor of outcome for papillary thyroid cancer? Ann Surg Oncol, 13, 1524-8.

Cisco R, Shen W, Gosnell J (2012). Extent of surgery for papillary thyroid cancer: preoperative imaging and role of prophylactic and therapeutic neck dissection. Curr Treat Options Oncol, 13, 1-10.

Cooper D, Doherty G, Haugen B, et al (2009). Revised American Thyroid Association management guidelines for patients with thyroid nodules and differentiated thyroid cancer. Thyroid, 19, 1167-214.

Dralle H (2012). Postoperative hypoparathyroidism: central neck dissection is a significant risk factor, Chirurg, 83, 1082 .

Dralle H (2013). Prophylactic central lymph node dissection improves prognosis for papillary thyroid cancer, Chirurg, 84, 149 .

Fritze D, Doherty G (2010). Surgical management of cervical lymph nodes in differentiated thyroid cancer. Otolaryngol Clin N Am, 43, 285-300.

Giordano D, Valcavi R, Thompson G, et al (2012). Complications of central neck dissection in patients with papillary thyroid carcinoma: results of a study on 1087 patients and review of the literature, Thyroid, 22, 911-17.

Hay I, Bergstralh E, Grant C, et al (1999). Impact of primary surgery on outcome in 300 patients with pathologic tumornode metastasis stage III papillary thyroid carcinoma treated at one institution from 1940 through. Surgery, 126, 1173-81; 
Ihab Samy Fayek

discussion 1181-2.

Hughes D, Doherty G (2011). Central neck dissection for papillary thyroid cancer. Cancer Control, 18, 83-8.

Ito Y, Miyauchi A (2007). Lateral and mediastinal lymph node dissection in differentiated thyroid carcinoma: indications, benefits, and risks. World J Surg, 31, 905-15.

Laura Y, Mark A, Anthony J, et al (2013). Level VII is an important component of central neck dissection for papillary thyroid cancer. Ann Surg Oncol, 20, 2261-5.

Leboulleux S, Rubino C, Baudin E, et al (2005). Prognostic factors for persistent or recurrent disease of papillary thyroid carcinoma with neck lymph node metastases and/or tumor extension beyond the thyroid capsule at initial diagnosis. $J$ Clin Endocrinol Metab, 90, 5723-9.

Machens A, Dralle H (2009). Prediction of mediastinal lymph node metastasis in papillary thyroid cancer. Ann Surg Oncol, 16, 171-6.

Mazzaferri E, Doherty G, Steward D (2009). The pros and cons of prophylactic central compartment lymph node dissection for papillary thyroid carcinoma, Thyroid, 19, 683-9.

Mulla M (2012). Central cervical lymph node metastases in papillary thyroid cancer: a systematic review of imagingguided and prophylactic removal of the central compartment. Clin Endocrinol (Oxf), 76,131-6.

Popadich A, Levin O, Lee J, et al (2011). A multicenter cohort study of total thyroidectomy and routine central lymph node dissection for cN0 papillary thyroid cancer. Surgery, 150, 1048-57.

Roh J, Kim J, Park C (2008). Central cervical nodal metastasis from papillary thyroid microcarcinoma: pattern and factors predictive of nodal metastasis. Ann Surg Oncol, 15, 2482-6.

Siegel R, Naishadham D, Jemal A (2013). Cancer statistics. CA Cancer J Clin, 63, 11-30.

Sobin L, Gospodarowicz M, Wittekind C (Eds.), (2010). UICC International union against cancer. TNM classification of malignant tumors, seventh ed., Wiley, West Sussex, UK, 58-62.

Steinmuller T, Klupp J, Rayes N, et al (2000). Prognostic factors in patients with differentiated thyroid carcinoma. Eur $J$ Surg, 166, 29-33.

Wang S, Zhao W, Wang W, et al (2013). Clinical features and prognosis of patients with benign thyroid disease accompanied by an incidental papillary carcinoma. Asian Pac J Cancer Prev, 14, 707-11.

White M, Gauger P, Doherty G, (2007). Central lymph node dissection in differentiated thyroid cancer. World J Surg, 31, 895-904.

Yu W, Tao S, Zhang N (2012). Is level V dissection necessary for low-risk patients with papillary thyroid cancer metastasis in lateral neck levels II, III, and IV. Asian Pac J Cancer Prev, 13, 4619-22. 